\title{
HIGH PRECISION COMPUTATION OF A CONSTANT IN THE THEORY OF TRIGONOMETRIC SERIES
}

\author{
J. ARIAS DE REYNA AND J. VAN DE LUNE
}

\begin{abstract}
Using the bisection as well as the Newton-Raphson method, we compute to high precision the Littlewood-Salem-Izumi constant frequently occurring in the theory of trigonometric sums.
\end{abstract}

\section{INTRODUCTION}

In Zygmund [15. p. 192] we read that there exists a number $\alpha_{0} \in(0,1)$ such that for each $\alpha \geq \alpha_{0}$ the partial sums of the series $\sum_{n=1}^{\infty} n^{-\alpha} \cos (n x)$ are uniformly bounded below, whereas for $\alpha<\alpha_{0}$ they are not. It is also shown there that $\alpha_{0}$ is the unique solution of the equation

$$
\int_{0}^{3 \pi / 2} u^{-\alpha} \cos u d u=0 \quad(0<\alpha<1) .
$$

(The uniqueness of $\alpha_{0}$ will also follow from our analysis in Section 4.)

In this journal ([5], [8] and [14) we find three short papers dealing with the numerical computation of this critical constant. In the first-mentioned paper the method of computation was not revealed. The result $0.30483<\alpha_{0}<0.30484$ appears to be incorrect in the third decimal (which was also observed in 8 and [14]). In the second paper, by conventional numerical quadrature, it was (correctly) found that $0.308443<\alpha_{0}<0.308444$. In the third paper, using differencing and making use of ordinary interpolation techniques, it was announced that (to $15 \mathrm{D}$ ) $\alpha_{0}=0.308443779561985$, which, as we will see, comes quite close to the true solution of (1.1).

The main object of this note is to present some simple elementary procedures for a high precision computation of $\alpha_{0}$.

Although we will not tackle (1.1) by any integral approximating procedure, anyone persisting to do so might consider first removing the singularity of the integrand in (1.1) at $u=0$ by integrating by parts, yielding the equivalent equation

$$
F(\alpha):=\int_{0}^{3 \pi / 2} u^{1-\alpha} \sin u d u=0 \quad(0<\alpha<1) .
$$

We might solve (1.1) by directly substituting the power series for $\cos u$. However, instead, we will tackle (1.2) by directly substituting the power series for $\sin u$,

Received by the editor July 28, 2008 and, in revised form, September 21, 2008.

2000 Mathematics Subject Classification. Primary 42-04, 26D05; Secondary 11Y60.

Key words and phrases. Trigonometric sums, Littlewood-Salem-Izumi constant, High precision computation.

The first author was supported by MCI Grant MTM2006-05622. 
yielding the equivalent equation

$$
\int_{0}^{3 \pi / 2} u^{1-\alpha} \sum_{k=0}^{\infty}(-1)^{k} \frac{u^{2 k+1}}{(2 k+1) !} d u=\sum_{k=0}^{\infty} \frac{(-1)^{k}}{(2 k+1) !} \int_{0}^{3 \pi / 2} u^{2 k+2-\alpha} d u=0
$$

(interchanging $\sum$ and $\int$ being permitted here by uniform convergence) or

$$
\sum_{k=0}^{\infty} \frac{(-1)^{k}}{(2 k+1) !} \frac{\left(\frac{3 \pi}{2}\right)^{2 k+3-\alpha}}{2 k+3-\alpha}=0 \quad(0<\alpha<1)
$$

which, in its turn, is clearly equivalent to

$$
G(\alpha):=\sum_{k=0}^{\infty} \frac{(-1)^{k}}{(2 k+1) !} \frac{\left(\frac{3 \pi}{2}\right)^{2 k}}{2 k+3-\alpha}=0 \quad(0<\alpha<1) .
$$

Note that $F(\alpha)$ and $G(\alpha)$ differ only by a positive factor:

$$
G(\alpha)=\left(\frac{3 \pi}{2}\right)^{\alpha-3} F(\alpha)=\frac{2}{3 \pi} \int_{0}^{1} v^{1-\alpha} \sin \left(\frac{3 \pi}{2} v\right) d v
$$

\section{Error analysis For $G(\alpha)$ AND $G^{\prime}(\alpha)$}

In order to compute $G(\alpha)$ sufficiently accurately we make the following error analysis. We will make use of the following simple and well-known

Lemma 2.1. If $a_{M+1}>a_{M+2}>a_{M+3}>\cdots>0$ and $\lim _{k \rightarrow \infty} a_{k}=0$, then the alternating series $\sum_{k=M+1}^{\infty}(-1)^{k} a_{k}$ converges and its sum $S$ satisfies $|S|<a_{M+1}$.

We can now easily show that when truncating (1.4) after $M$ terms we commit an (absolute) error $<\frac{(3 \pi / 2)^{2 M+2}}{(2 M+4) !}$. Writing

$$
a_{k}=\frac{\left(\frac{3 \pi}{2}\right)^{2 k}}{(2 k+1) !(2 k+3-\alpha)}
$$

we clearly have $a_{k}>0, \lim _{k \rightarrow \infty} a_{k}=0$ and

$$
\frac{a_{k+1}}{a_{k}}=\frac{\left(\frac{3 \pi}{2}\right)^{2}}{(2 k+2)(2 k+3)} \frac{2 k+3-\alpha}{2 k+5-\alpha}<\frac{23}{42}<1 \quad \text { for } k \geq 2
$$

so that the lemma applies. Hence

$$
\left|\sum_{k=M+1}^{\infty}(-1)^{k} \frac{\left(\frac{3 \pi}{2}\right)^{2 k}}{(2 k+1) !(2 k+3-\alpha)}\right|<a_{M+1}<\frac{\left(\frac{3 \pi}{2}\right)^{2 M+2}}{(2 M+4) !} \quad \text { for } M \geq 1,
$$

proving our claim. (Note that we used that $0<\alpha<1$.)

In a similar way it is easily seen that the same $M$ yields an even smaller error when

$$
G^{\prime}(\alpha)=\sum_{k=0}^{\infty} \frac{(-1)^{k}}{(2 k+1) !} \frac{\left(\frac{3 \pi}{2}\right)^{2 k}}{(2 k+3-\alpha)^{2}}
$$

is truncated after $M$ terms. 


\section{THE BISECTION PROGRAM}

We first present a program (for Mathematica Version 4.2) using bisection of the $\alpha$-interval $[0,1]$. This very robust procedure needs no further justification. The result is (accurate to $130 \mathrm{D}$ ):

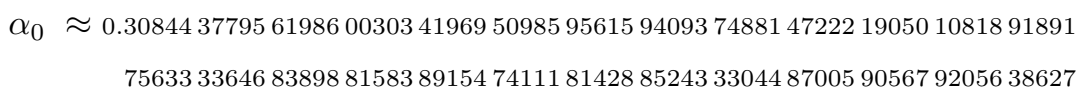

\section{JUSTificAtion OF THE APPLICATION OF THE \\ NEWTON-RAPHSON METHOD}

In order to solve the equation $G(\alpha)=0$ we will now apply the much faster Newton-Raphson method. We will show that (1.4) can also be used for this purpose.

Our justification for applying this method here is based on the following three observations:

Observation 1. $G(0)<0<G(1)$.

Proof. From (1.5) it easily follows that

$$
G(0)=-\left(\frac{2}{3 \pi}\right)^{3} \quad \text { and } \quad G(1)=\left(\frac{2}{3 \pi}\right)^{2} .
$$

Observation 2. $G^{\prime}(\alpha)>(2 / 3 \pi)^{3}$ for $0 \leq \alpha \leq 1$.

Proof. Since (with $\left.\operatorname{Si}(x)=\int_{0}^{x} \sin t / t d t\right)$

$$
G^{\prime}(0)=-\frac{2}{3 \pi} \int_{0}^{1} v \log v \sin \left(\frac{3 \pi}{2} v\right) d v=\frac{8\left(1+\operatorname{Si}\left(\frac{3 \pi}{2}\right)\right)}{27 \pi^{3}}>\left(\frac{2}{3 \pi}\right)^{3},
$$

our claim is an easy consequence of the following

Observation 3. $G^{\prime \prime}(\alpha)>0$ for $0 \leq \alpha \leq 1$.

Proof. Writing $c=\frac{3 \pi}{2}$ and $a_{k}=\frac{2 c^{2 k}}{(2 k+1) !(2 k+3-\alpha)^{3}}(>0)$ it follows from (1.4) that

$$
G^{\prime \prime}(\alpha)=\sum_{k=0}^{\infty}(-1)^{k} a_{k}=\left(a_{0}-a_{1}\right)+\sum_{k=1}^{\infty}\left(a_{2 k}-a_{2 k+1}\right) .
$$

Since

$$
\frac{a_{2 k+1}}{a_{2 k}}=\frac{c^{2}}{(4 k+2)(4 k+3)}\left(\frac{4 k+3-\alpha}{4 k+5-\alpha}\right)^{3}<\frac{23}{42} \cdot 1<1 \quad \text { for } \quad k \geq 1,
$$

we already find that $G^{\prime \prime}(\alpha)>a_{0}-a_{1}$.

So, it suffices to show that $a_{0} \geq a_{1}$, or, equivalently, that

$$
\frac{a_{1}}{a_{0}}=\frac{c^{2}}{3 !}\left(\frac{3-\alpha}{5-\alpha}\right)^{3} \leq 1 .
$$

Since $(0<) \frac{3-\alpha}{5-\alpha}=1-\frac{2}{5-\alpha}$ is decreasing (for $0 \leq \alpha \leq 1$ ) we need only check whether $\frac{c^{2}}{6}\left(\frac{3}{5}\right)^{3} \leq 1$. Since $c^{2}<23$ it suffices to observe that $\frac{23}{6} \frac{27}{125}=\frac{207}{250}<1$, and the proof is complete. 
From the previous proof it is clear that $\sum_{k=0}^{\infty}(-1)^{k} a_{k}$ is an alternating series in the sense of Lemma 2.1. Hence, we might use a similar error estimate as given before for $G(\alpha)$ and $G^{\prime}(\alpha)$.

The upshot of all this is that (for $0 \leq \alpha \leq 1$ )

(a) $G(\alpha)$ changes sign, (b) $G(\alpha)$ is strictly increasing, (c) $G(\alpha)$ is strictly convex.

It is well known that these conditions are sufficient for a rigorous application of the Newton-Raphson method to solve our equation $G(\alpha)=0$.

Starting on the large side with $\alpha=\frac{31}{100}$, the program in Section 5 yields the solution $\alpha_{0}$ presented there (accurate to $1120 \mathrm{D}$ ).

\section{The NEWTON-RAPHSON PROGRAM}

Considerably more efficient than the bisection procedure is the Newton-Raphson method. The theoretical justification was given in the previous section.

Starting with $\alpha=\frac{31}{100}$ we find after 10 iterations that

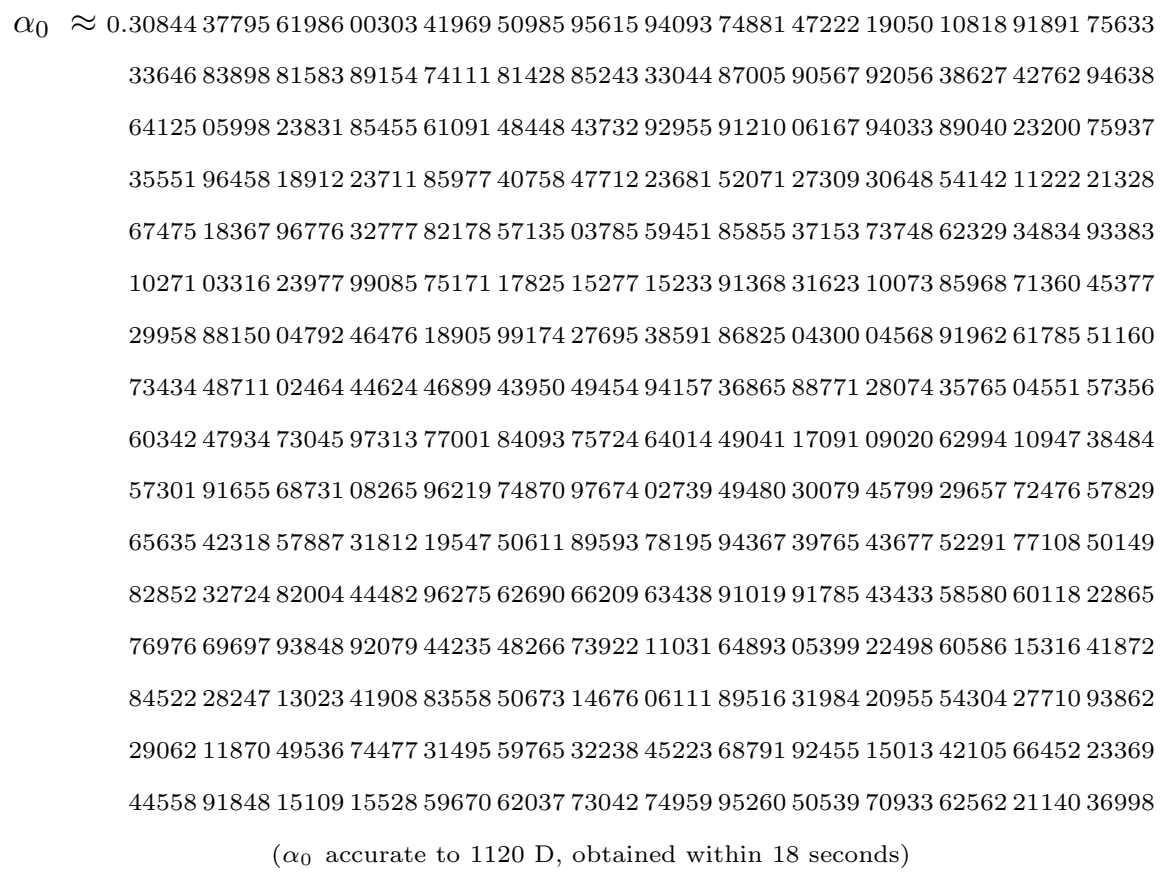

Without any economization of our Newton-Raphson program, the computation of $\alpha_{0}$ to $5000 \mathrm{D}$ (requiring 12 iterations) took less than 20 minutes on a Toshiba laptop - 2 GB RAM - $3.2 \mathrm{MHz}$.

\section{ACKNOWLEDGEMENTS}

We want to thank the referee for his valuable suggestions to simplify our original derivations in Sections 2 and 4.

Our thanks are also due to Professors R. A. Askey, S. R. Finch and S. Koumandos for pointing out various closely related references to us.

Note. Zygmund [15, p. 379] writes that the origin of the defining property for $\alpha_{0}$ is to be found in an unpublished result of Littlewood and Salem, and that the equation 
defining $\alpha_{0}$ is due to S. Izumi. This justifies calling it the Littlewood-Salem-Izumi constant.

However, the earliest paper where we detected this constant is [10].

For additional information on $\alpha_{0}$ we recommend [2] and [9. $\alpha_{0}$ also plays a role in some theorems about positive trigonometric series with general coefficients: [4], 6] and [7, and in theorems about the positivity of some sums of orthogonal polynomials: 12 .

Our method can be extended to apply to similar constants. For example, some open problems are mentioned in [1, [3] and [13.

\section{REFERENCES}

1. R. Askey, J. Steinig, Some positive trigonometric sums, Trans. Amer. Math. Soc. 187 (1974), 295-307. MR0338481 (49:3245)

2. R. Askey, Orthogonal Polynomials and Special Functions (CBMS-NSF Regional Conference Series in Applied Mathematics), Society for Industrial and Applied Mathematics, 1987. MR 0481145 (58:1288)

3. R. Askey, Problems which interest and/or annoy me, J. Comput. Appl. Math. 48 (1993), 3-15. MR.1246848 (94j:33005)

4. A. S. Belov, Coefficients of trigonometric cosine series with nonnegative partial sums (in Russian), Translated in Proc. Steklov Inst. Math. (1992) 1, 1-18. Theory of functions (in Russian) (Amberd, 1987). Trudy Mat. Inst. Steklov 190 (1989), 3-21. MR1005335 (90m:42005)

5. R. P. Boas Jr. and C. Klema, A constant in the theory of trigonometric series, Math. Comp. 18 (1964), 674. MR.0176283 (31:558)

6. G. Brown, K. Wang, D. C. Wilson, Positivity of some basic cosine sums, Math. Proc. Cambridge Philos. Soc. 114 (1993), 383-391. MR1235986 (94h:42013)

7. G. Brown, F. Dai, K. Wang, On positive cosine sums, Math. Proc. Camb. Phil. Soc. 142 (2007), 219-232. MR:2314596 (2008e:42014)

8. R. F. Church, On a constant in the theory of trigonometric series, Math. Comp. 19 (1965), 501.

9. S. R. Finch, Mathematical Constants, Cambridge University Press, Cambridge, UK, 2003. MR2003519 (2004i:00001)

10. K. Grandjot, V. Jarnik, E. Landau, J. E. Littlewood, Bestimmung einer absoluten Konstanten aus der Theorie der trigonometrischen Reihen, Annali di Mat. (4) 6 (1929), 1-7. MR1553122

11. J. Keane, Estimating Brown-Wang $B$ and Zygmund $R$ constants, unpublished note (2000).

12. S. Koumandos, S. Ruscheweyh, Positive Gegenbauer polynomial sums and applications to starlike functions, Constr. Approx. 23 (2006), 197-210. MR2186305 (2007b:42001)

13. S. Koumandos, S. Ruscheweyh, On a conjecture for trigonometric sums and starlike functions, J. Approx. Theory 149 (2007), 42-58. MR2371613

14. Y. L. Luke, W. Fair, G. Coombs and R. Moran, On a constant in the theory of trigonometric series, Math. Comp. 19 (1965), 501-502.

15. A. Zygmund, Trigonometric Series, Cambridge University Press (1988) Vol I. MR.933759 (89c:42001)

Facultad de Matemáticas, Universidad de Sevilla, Apdo. 1160, 41080-Sevilla, Spain

E-mail address: arias@us.es

Langebuorren 49, 9074 CH Hallum, The Netherlands (formerly at CWi, Amsterdam)

E-mail address: j.vandelune@hccnet.nl 